\title{
The effects of strength training on isometric force production symmetry in recreationally trained males
}

\author{
Caleb D. Bazyler, Chris A. Bailey, Chieh-Ying Chiang, Kimitake Sato, Michael H. Stone
}

Objectives: The purpose of this investigation was to determine what effect a bilateral strength training regimen has on isometric force production symmetry and if changes in force production symmetry can be accounted for by differences in pre-intervention strength levels.

Design: Sixteen recreationally trained males (1-RM squat: $146.8 \pm 23.0 \mathrm{~kg}$.) were assigned to two groups for the 7-week training intervention: strong (S) and weak (W) based on pre-training squat isometric peak force allometrically scaled (IPFa) at $120^{\circ} \mathrm{knee}$ angle.

Methods: Subjects completed a 7-week training intervention following a block-periodized model and were tested on measures of dynamic (1RM squat) and isometric (isometric squat at $90^{\circ}$ and $120^{\circ}$ knee angle) strength pre- and post-intervention. The degree of bilateral lower limb asymmetry was calculated as a percentage where $0 \%$ symmetry index (SI) indicates perfect symmetry on the isometric squat.

Results: ANCOVA results showed no statistical difference between groups for all dependent variables when pre-intervention IPFa $120^{\circ}$ scores were used as the covariate. Paired t-tests results showed both groups statistically improved 1RM squat and IPFa $120^{\circ}(p<0.05)$. IPFa $120^{\circ}$ SI decreased statistically from pre-training in the $\mathrm{W}$ group $(p=0.03)$. Independent t-test results showed the W group had statistically larger pre-intervention SI scores for IPFa $90^{\circ}(p=0.045)$ and IPFa $120^{\circ}(p=0.007)$; however this difference was no longer present following strength training. There was a strong inverse relationship between pooled IPFa $120^{\circ}$ and IPFa $120^{\circ}$ SI $(r=-0.64, p=0.004)$.

Conclusions: The findings of the current study support the notion that weaker individuals can augment lower limb symmetry with strength training. The same does not seem to be true for stronger individuals who already have a low symmetry index score. These findings indicate that strength training improves force production symmetry in relatively weak males, which may be important for bilateral tasks and injury potential reduction.

(Journal of Trainology 2014;3:6-10)

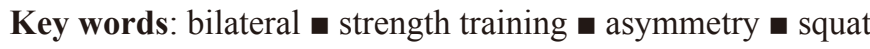

\section{INTRODUCTION}

Recently, limb asymmetry in sport has gained interest among coaches and athletes and has become a more prevalent research topic. ${ }^{1,2}$ Specifically, the relationships between force production symmetry, injury, and performance seem to be a common interest..$^{3-5}$ The lack of ability to produce symmetrical movement patterns and the inability to produce force symmetrically have been indicated as risk factors for injury, but currently there remains some doubt due to a lack of direct evidence to support this claim. ${ }^{6,7}$

The role symmetry plays on performance has not been researched as extensively. Yoshioka and colleagues (4) completed a computer simulation study which compared a symmetrical and an asymmetrical model of the lower limbs during jumping tasks. The models were equated for strength, but the distribution of the strength between limbs differed. Their results showed that the stronger limb of the asymmetrical model would make up the difference in the weaker limb and jump height would not be affected as a result. Bailey et al. ${ }^{5}$ reported different findings in a study measuring symmetry of an isometric mid-thigh pull. Their findings indicate a negative relationship exists between isometric peak force symmetry and both jump height and peak power during counter movement and static jumps. Thus, the relationship between strength symmetry and performance requires additional research.

Even less research has examined the role of strength training to reduce asymmetry. If symmetrical force production and strength capabilities are desired qualities, one may wish to reduce strength asymmetry by strength training. Therefore, the purpose of this study was to determine what effect a bilateral strength training regimen has on isometric force production symmetry and if changes in force production symmetry can be accounted for by differences in pre-intervention strength levels.

Received January 24, 2014; accepted February 20, 2014

From the Department of Exercise and Sport Science, Center of Excellence for Sport Science and Coach Education, East Tennessee State University, Johnson City, TN, USA (C.D.B, C.A.B., C-Y.C., K.S., M.H.S.)

Communicated by Takashi Abe, PhD

Correspondence to Mr. Caleb D. Bazyler, M.A., CSCS., Center of Excellence for Sport Science and Coach Education, East Tennessee State University, P.O. Box 70654, Johnson City, TN 37614-1701, USA. Email: bazyler@goldmail.etsu.edu

Journal of Trainology 2014:3:6-10 @2012 The Active Aging Research Center http://trainology.org/ 


\section{Subjects \\ METHODS}

Subjects recruited were 20 recreationally trained college aged males with at least one year of resistance training experience on the squat $(\geq 1.3 \mathrm{x}$ body mass). Only18 subjects were included in the data analysis; one withdrew due to injury and another was excluded as an outlier (produced values greater than twice the standard deviation of the pooled mean). The one repetition maximum (1-RM) squat indicated that the subjects were moderately trained: pre-training 1-RM squat: $146.8 \pm$ $23.0 \mathrm{~kg}$., squat to body mass ratio: $1.68 \pm 0.22 .^{8-10}$ Subjects were ranked based on pre-training squat isometric peak force allometrically scaled (IPFa) identified at knee angle of $120^{\circ}$ and divided into 2 groups, weak (W) and strong (S). Allometric scaling is based on the surface law, which states that as the volume of a body increases, its surface area reduces in proportion. ${ }^{11}$ Thus, IPFa was calculated by dividing peak force by body mass raised to the 0.67 power $\left(\mathrm{N} / \mathrm{kg}^{0.67}\right)$. Nine subjects in the W group (age $20.8 \pm 2.0$ years, height $176.4 \pm$ $6.3 \mathrm{~cm}$, body mass $84.9 \pm 10.9 \mathrm{~kg}$ ) and nine subjects in the $\mathrm{S}$ group (age $20.7 \pm 1.9$ years, height $177.6 \pm 8.1 \mathrm{~cm}$, body mass $86.1 \pm 8.9 \mathrm{~kg}$ ) completed the study. Throughout the study, subjects were instructed not to participate in physical activity 24 hours prior to testing or training sessions. Prior to participating, all subjects completed a health history questionnaire and signed an informed consent that was approved by the university's Institutional Review Board. Although subjects were not specifically questioned about previous leg injuries, they were required to report injuries that could be exacerbated by participating in the study. Both groups performed back squats 2 $\mathrm{d} \cdot \mathrm{wk}^{-1}$ for 12 weeks with a minimum of 48 hours rest between training sessions. Dynamic and isometric strength were measured pre- and post-intervention via 1-RM and isometric squat, respectively.

\section{Training}

After eligibility was determined by 1-RM squat testing $(\geq 1.3$ $\mathrm{x}$ body weight), subjects trained $2 \mathrm{~d} \cdot \mathrm{wk}^{-1}$ for 3 weeks in a strength-endurance phase to equilibrate the training program for all subjects. During this phase, subjects were familiarized with isometric squats to minimize learning effects and to record bar heights and knee angles for subsequent testing. Table I describes the 12-week training program and testing sessions. Subjects were required to complete $>80 \%$ of the programmed volume load to be included in the data analysis.

Each training session began with a dynamic warm-up using only body weight, followed by warm-up sets on the squat. Groups followed a block-periodized model with heavy and light days within each microcycle to manage fatigue. ${ }^{12,13}$ Load for squat and partial-squat was calculated using percentage of pre-intervention 1-RM. All training sessions were supervised to ensure correct technique and safety.

\section{Testing Procedures}

Anthropometrics and 1-RM squat were measured at the beginning of week 4 and 12 dynamic testing sessions. IPFa at a $120^{\circ}$ knee angle, IPFa at a $90^{\circ}$ knee angle, and symmetry index (SI) scores for IPFa $120^{\circ}$, IPFa $90^{\circ}$ were assessed during the isometric testing session, which occurred 72-96 hours after dynamic testing.

\section{Dynamic Strength Assessment}

Once subjects arrived, anthropometrics were measured, followed by a dynamic warm-up. 1-RM squat protocol involved a progressive increase in load and decrease in reps per set. ${ }^{8}$ 1-RM squat attempts were selected with the goal of reaching their max in three attempts after warm-up. Four minutes of rest was given between each attempt. Back squat depth was determined as the top of the leg at the hip joint being below the knee. ${ }^{14}$

Table I: Strength Training Program

\begin{tabular}{lccccc}
\hline Phase & Week & Day 1 & $\% 1 \mathrm{RM}$ & Day 2 & $\%$ Reduction \\
\hline \multirow{2}{*}{ Strength-Endurance } & 1 & $4 \times 8$ & $75-80 \%$ & $3 \times 8$ & $10-15 \%^{\mathrm{a}}$ \\
and Familiarization & 2 & $4 \times 8$ & $77.5-82.5 \%$ & $3 \times 8$ & $10-15 \%$ \\
& 3 & $4 \times 8$ & $80-85 \%$ & $3 \times 8$ & $10-15 \%$ \\
\hline Pre-Testing & 4 & Dynamic & & Isometric & \\
\hline \multirow{2}{*}{ Strength Phase 1 } & 5 & $6 \times 5$ & $85-87 \%$ & $6 \times 5$ & $10-15 \%$ \\
& 6 & $6 \times 5$ & $86-88 \%$ & $6 \times 5$ & $10-15 \%$ \\
\hline De-Load & 7 & $6 \times 5$ & $87-89 \%$ & $6 \times 5$ & $15-20 \%$ \\
\hline & 8 & $6 \times 3$ & $75 \%$ & $6 \times 3$ & $15-20 \%$ \\
Strength Phase 2 & 9 & $6 \times 3$ & $88-90 \%$ & $6 \times 3$ & $10-15 \%$ \\
& 10 & $6 \times 3$ & $89-91 \%$ & $6 \times 3$ & $10-15 \%$ \\
\hline Post-Testing & 11 & $6 \times 3$ & $90-92 \%$ & $6 \times 3$ & $15-20 \%$ \\
\hline
\end{tabular}

${ }^{\mathrm{a}}$ represents $\%$ reduction in load from Day 1 


\section{Isometric Strength Assessment}

Kinetic variables were measured on $0.45 \mathrm{~m}$ x $0.91 \mathrm{~m}$ dual force platforms affixed side by side (Rice Lake, WI) sampling at $1,000 \mathrm{~Hz}$ inside a custom designed power rack that allows fixation of the bar at any height, as described previously. ${ }^{15}$ Subjects performed a dynamic warm-up followed by two warm-up attempts at $50 \%$ and $75 \%$ of perceived maximal effort at $90^{\circ}$ angle of the knee. After the two-minute rest period, two maximal efforts were performed with 3 minutes rest in between trials. The bar was placed across the back in the same position used in training and placed against two metal stops to prevent upward movement (Figure 1). The same assistant recorded knee angle and bar height for all testing sessions. The tester instructed subjects to push as fast and as hard as possible. ${ }^{16}$ The tester shouted 'push' and participants pushed maximally into the ground until peak force was reached when the tester shouted 'stop' to end the test. After completing testing at $90^{\circ}$ subjects were given 5 minutes rest and the same protocol was repeated at a $120^{\circ}$ knee angle. Subjects were tested at the same time of the day for both test days. ${ }^{17}$ SI was calculated using the following equation:

\section{$S I=100 \% \times($ Larger value - smaller value $) /$ sum of values}

(Shorter, Polk \& Rosengren et al. ${ }^{18}$; Sato \& Heise ${ }^{1}$ ).

The resultant is a percentage where $0 \%$ indicates perfect symmetry and the level of asymmetry increases as the value gets further away from zero. The force-time curve data were

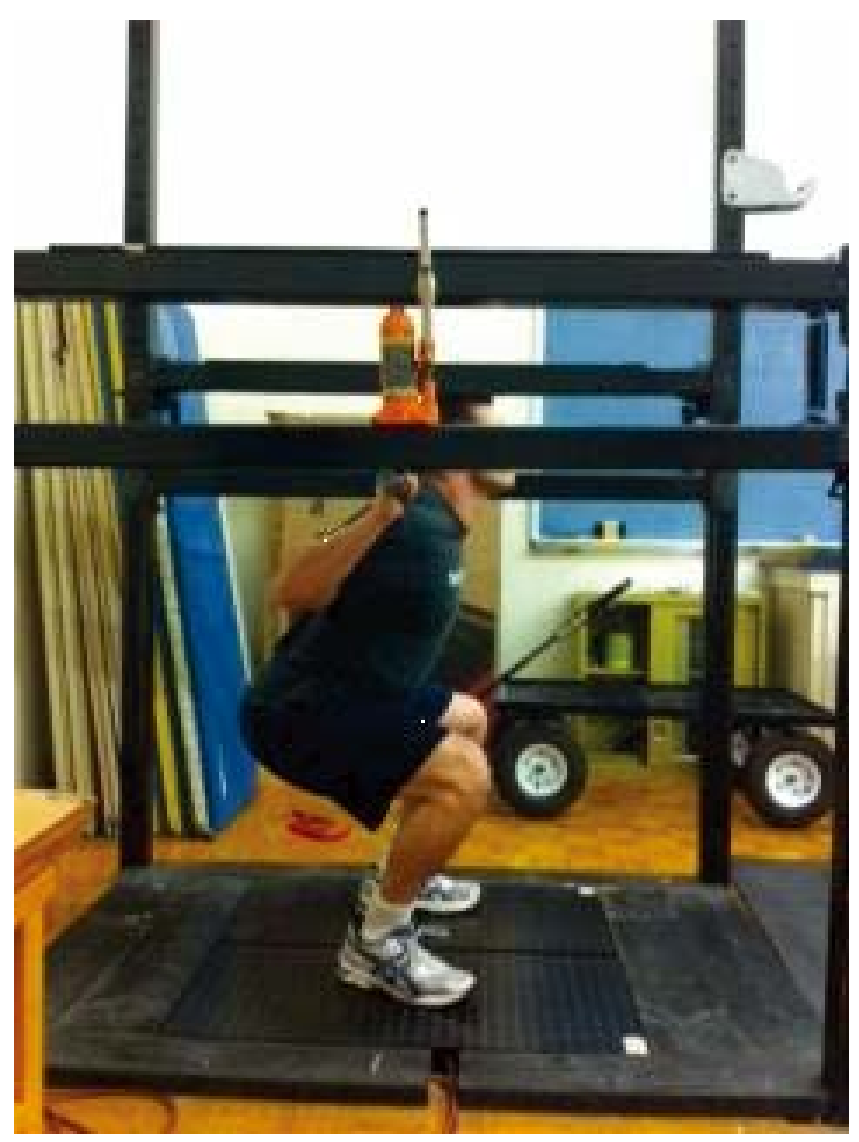

Figure 1 Isometric Squat at $90^{\circ}$ knee flexion smoothed using an 11-point moving average (all data points equally weighted) and analyzed with Labview software (ver. 2010, National Instruments, Austin, TX, USA). The average of two attempts on the isometric squat at 90 and $120^{\circ}$ were used for analysis.

\section{Statistical Analysis}

A Shapiro-Wilks normality test was used to determine if the data were normally distributed. A Levene-test was used to determine homogeneity of variance. Intra-class correlation coefficients (ICC) were calculated to determine test-retest reliability. As a result of the statistical difference between groups for pre-intevention IPFa $120^{\circ}$ scores, a univariate analysis of covariance (ANCOVA) was used to assess differences between the two groups post-intervention for all dependent variables. Paired sample t-test and independent samples t-test were calculated to determine within and between group differences for dependent variables. A Pearson's product-moment correlation was used to assess the relationship between dependent variables. For all tests the alpha level was set at $p<0.05$. SPSS software version 21 was used to perform all statistical analyses (IMB Co., NY, USA).

\section{RESULTS \\ Dynamic Strength Assessment \\ 1-RM Squat}

After adjusting for pre-intervention scores, ANCOVA results showed no statistical difference between groups for 1-RM squat post-intervention. The mean values for 1-RM squat in $\mathrm{S}$ increased from $167.57 \pm 26.44$ to $175.99 \pm 26.44 \mathrm{~kg}(\mathrm{p}<0.001$, $+5.0 \%)$ and in the $\mathrm{W}$ from $137.84 \pm 19.10$ to $146.91 \pm 17.67$ $\mathrm{kg}(\mathrm{p}<0.001,+6.6 \%)$.

\section{Isometric Strength Assessment Isometric Squat Peak Force Scaled Symmetry Index}

After adjusting for pre-intervention scores, ANCOVA results showed no statistical difference between groups for IPFa $90^{\circ}$ SI or IPFa $120^{\circ}$ SI post-intervention. Paired t-test results indicated that IPFa $120^{\circ}$ SI decreased statistically from pre-training in the $\mathrm{W}$ group $(\mathrm{p}=0.03)$; however, IPFa $90^{\circ} \mathrm{SI}$ showed no statistical change in either group. Independent t-test results showed a statistical difference between groups pre-training for IPFa $120^{\circ} \mathrm{SI}(\mathrm{p}=0.007)$ and for IPFa $90^{\circ} \mathrm{SI}(\mathrm{p}=0.045)$ with the $\mathrm{W}$ group being statistically greater than the $\mathrm{S}$ group; these differences between groups were not present following strength training. IPFa $120^{\circ}$ statistically increased in both groups $(\mathrm{p}<0.05)$, whereas only the $\mathrm{S}$ group statistically improved IPFa $90^{\circ}(\mathrm{p}=0.01)$. There was also a strong inverse relationship between IPFa $120^{\circ}$ and IPFa $120^{\circ}$ SI $(r=-0.64, p=0.004)$. Testretest reliability using ICC for IPFa $90^{\circ}$ and $120^{\circ}$ was 0.98 and 0.98 , respectively. Mean and standard deviation for dependent variables are listed in Table II. 
Table II: Changes in Dependent Variables

\begin{tabular}{|c|c|c|c|c|}
\hline & \multicolumn{2}{|c|}{ Strong } & \multicolumn{2}{|c|}{ Weak } \\
\hline & Pre & Post & Pre & Post \\
\hline IPF a $\left(\mathrm{N} / \mathrm{Kg}^{0.67}\right) 120^{\circ}$ & $227.96 \pm 17.45 \S \S$ & $239.95 \pm 19.24 * \S \S$ & $172.85 \pm 19.86$ & $186.54 \pm 29.28^{*}$ \\
\hline IPF a $\left(\mathrm{N} / \mathrm{Kg}^{0.67}\right) 90^{\circ}$ & $115.75 \pm 11.14 \S$ & $121.54 \pm 7.34 * \S$ & $106.77 \pm 10.02$ & $108.29 \pm 9.78$ \\
\hline IPF a $\left(\mathrm{N} / \mathrm{Kg}^{0.67}\right) 120^{\circ} \mathrm{SI}$ & $1.89 \pm 1.09 \S$ & $2.22 \pm 1.22$ & $3.91 \pm 1.75$ & $1.89 \pm 1.50^{*}$ \\
\hline IPF a $\left(\mathrm{N} / \mathrm{Kg}^{0.67}\right) 90^{\circ} \mathrm{SI}$ & $2.23 \pm 1.67 \S$ & $2.58 \pm 2.01$ & $4.60 \pm 4.26$ & $3.95 \pm 5.06^{*}$ \\
\hline 1-RM Squat (Kg) & $167.57 \pm 26.44 \S$ & $175.99 \pm 26.44 * \S$ & $137.84 \pm 19.10$ & $146.91 \pm 17.67 *$ \\
\hline
\end{tabular}

*statistically different between Pre and Post, $\S$ statistically different between groups, ${ }^{*} \mathrm{P}<0.05 ;{ }^{*} * \mathrm{P}<0.01, \S \mathrm{P}<0.05$, $\S \S \mathrm{P}<0.001$. IPFa: Isometric peak force allometrically scaled

\section{DISCUSSION}

The findings of this study indicate that there is strong inverse relationship between isometric strength and bilateral lower limb asymmetry and that weaker individuals can augment lower limb symmetry with strength training. Although changes in lower limb asymmetry as a result of strength training have not been researched extensively, some research findings support that strength imbalances increase injury risk. ${ }^{3,6,19}$ However, there is still a lack of consensus on what a normal imbalance is and how much deviation is acceptable. ${ }^{20}$ The current findings indicate that strength training can decrease lower body muscle imbalances, agreeing with the available previous research. Golik-Peric and colleagues found that 4 weeks of isoinertial and isokinetic training in athletes improved ipsilateral muscle symmetry by increasing the concentric hamstring to quadriceps torque ratio (conH/Q). In their study, athletes were divided into an isokinetic and isoinertial training group. After completing a 4-week training program, the isokinetic and isoinertial groups increased the conH/Q ratio by $25.4 \%$ and $13.0 \%$ on the right lower limb, respectively. ${ }^{21}$ In comparison, the results of this paper showed a $51.5 \%$ reduction in contralateral lower limb asymmetry in the weaker group at a $120^{\circ}$ knee angle. The larger percent changes in our study are likely related to the longer duration of the training program (7-week intervention). Similarly, Impellizzeri and colleagues (2007) showed a $56.5 \%$ decrease in bilateral vertical jump force asymmetry following anterior cruciate ligament reconstruction and 7-9 weeks of physical therapy programs. ${ }^{22}$

The subjects in the current study were not collegiate athletes, but they did exhibit strength levels equivalent to and greater than previous research with some athletes. ${ }^{8-10}$ Although both groups statistically improved 1-RM squat only the weaker statistically decreased strength asymmetry, indicating that gains in bilateral strength may only decrease lower limb asymmetries to a point. Whether or not the decrease in asymmetry in the weaker group was directly responsible, independent of bilateral gains, for enhanced performance on the isometric squat or 1-RM squat is beyond the scope this study. However, it can be surmised that strength training improves force production symmetry in relatively weak males, which may be important for bilateral tasks and injury potential reduction.

\section{CONCLUSION}

The findings of the current study support the notion that as lower limb strength increases there is a concomitant decrease in asymmetry in weaker individuals. The same does not seem to be true for stronger individuals who already have a low symmetry index score. The decreases in asymmetry found in the weaker group may have implications for bilateral movements requiring symmetrical force production. Future research should address what is a normative symmetry index score, do larger asymmetries result in decrements in performance and increased injury risk, if so, identifying the magnitude of asymmetry can be an important issue for further investigation.

\section{ACKNOWLEDGMENTS}

The authors wish to confirm that there is no conflict of interest associated with this publication and that there has been no financial support for this work that could have influenced its outcome. The authors would like to thank the ETSU sports science students for assisting with the strength training and data collection.

\section{REFERENCES}

1. Sato K, Heise GD. Influence of weight distribution asymmetry on the biomechanics of a barbell back squat. J Strength Cond Res 2012; 26: $342-$ 349.

2. Benjanuvatra N, Lay BS, Alderson JA, Blanksby BA. Comparison of ground reaction force asymmetry in one- and two-legged countermovement jumps. J Strength Cond Res 2013; 27: 2700-2707.

3. Nadler SF, Malanga GA, Feinberg JH, Prybicien M, Stitik TP, DePrince M. Relationship between hip muscle imbalance and occurrence of low back pain in collegiate athletes: a prospective study. Am J Phys Med Rehabil 2001; 80: 572-577.

4. Yoshioka S, Nagano A, Hay DC, Fukashiro S. The effect of bilateral asymmetry of muscle strength on the height of a squat jump: a computer simulation study. J Sports Sci 2011; 29: 867-877.

5. Bailey, C. A., Sato, K., Alexander, R. P., Chiang, C. Y., \& Stone, M. H. Isometric force production symmetry and jumping performance in collegiate athletes. J Trainol 2013; 2: 1-5.

6. Kiesel K, Plisky PJ, Voight ML. Can Serious Injury in Professional Football be Predicted by a Preseason Functional Movement Screen? N Am J Sports Phys Ther 2007; 2: 147-158.

7. Zifchock RA, Davis I, Higginson J, McCaw S, Royer T. Side-to-side differences in overuse running injury susceptibility: a retrospective study. Hum Mov Sci 2008; 27: 888-902.

8. Zourdos MC, Kim JS. Physiological responses to two different models of 
daily undulating periodization in trained powerlifters. ProQuest 2012

9. Harris G, Stone M, O'Bryant H, Proulx C, Johnson R. Short-term performance effects of high power, high force, or combined weighttraining methods. J Strength Cond Res 2000; 14: 14-21.

10. Hoffman JR, Ratamess NA, Lkatt M, Faigenbaum AD, Ross RE, Tranchina NM, et al. Comparison between different offseason resistance training programs in Division III American college football players. $J$ Strength Cond Res 2009; 23: 11-19.

11. Schmidt-Nielsen K. Scaling: why is animal size so important? Cambridge University Press; 1984.

12. Painter KB, Haff GG, Ramsey MW, McBride J, Triplett T, Sands WA, et al. Strength gains: block versus daily undulating periodization weight training among track and field athletes. Int J Sports Physiol Perform 2012; 7: 161-169.

13. Stone M, Stone M, Sands W. Principles and practice of resistance training. Champaign, IL: Human Kinetics; 2007.

14. USAPL and IPF Administrators. USAPL rulebook and by-laws. 2001.

15. Beckham G, Mizuguchi S, Carter C, Sato K, Ramsey M, Lamont H, et al. Relationships of isometric mid-thigh pull variables to weightlifting performance. J Sports Med Phys Fitness 2013 Oct;53(5):573-581.

16. Holtermann A, Roeleveld K, Vereijken B, Ettema G. The effect of rate of force development on maximal force production: acute and training-related aspects. Eur J Appl Physiol 2007; 99: 605-613.

17. Häkkinen K, Pakarinen A, Alen M, Kauhanen H, Komi PV. Neuromuscular and hormonal responses in elite athletes to two successive strength training sessions in one day. Eur J Appl Physiol Occup Physiol 1988; 57: 133-139.

18. Shorter KA, Polk JD, Rosengren KS, Hsiao-Wecksler ET. A new approach to detecting asymmetries in gait. Clin Biomech 2008; 23: 459-467.

19. Knapik JJ, Bauman CL, Jones BH, Harris JM, Vaughan L. Preseason strength and flexibility imbalances associated with athletic injuries in female collegiate athletes. Am J Sports Med 1991; 19: 76-81.

20. Impellizzeri FM, Bizzini M, Rampinini E, Cereda F, Maffiuletti NA. Reliability of isokinetic strength imbalance ratios measured using the Cybex NORM dynamometer. Clin Physiol Funct Imaging 2008; 28: 113 119.

21. Golik-Peric D, Drapsin M, Obradovic B, Drid P. Short-Term Isokinetic Training Versus Isotonic Training: Effects on Asymmetry in Strength of Thigh Muscles. J Hum Kinet 2011; 30: 29-35.

22. Impellizzeri FM, Rampinini E, Maffiuletti N, Marcora SM. A vertical jump force test for assessing bilateral strength asymmetry in athletes. Med Sci Sports Exerc 2007; 39: 2044-2050. 